\title{
LAMINAR FILM CONDENSATION ON A HORIZONTAL PLATE IN A POROUS MEDIUM WITH SURFACE TENSION EFFECTS
}

Tong-Bou Chang

Assistant Professor, Department of Mechanical Engineering, Southern Taiwan University of Technology, 1, Nai-Tai Street, YungKang City, Tainan County, Taiwan, R.O.C, tbchang@mail.stut.edu.tw

Follow this and additional works at: https://jmstt.ntou.edu.tw/journal

Part of the Mechanical Engineering Commons

\section{Recommended Citation}

Chang, Tong-Bou (2005) "LAMINAR FILM CONDENSATION ON A HORIZONTAL PLATE IN A POROUS MEDIUM WITH SURFACE TENSION EFFECTS," Journal of Marine Science and Technology. Vol. 13: Iss. 4, Article 3.

DOI: $10.51400 / 2709-6998.2119$

Available at: https://jmstt.ntou.edu.tw/journal/vol13/iss4/3

This Research Article is brought to you for free and open access by Journal of Marine Science and Technology. It has been accepted for inclusion in Journal of Marine Science and Technology by an authorized editor of Journal of Marine Science and Technology. 
LAMINAR FILM CONDENSATION ON A HORIZONTAL PLATE IN A POROUS MEDIUM WITH SURFACE TENSION EFFECTS

Acknowledgements

This work was supported by the National Science Council of ROC: NSC93-2212-E-218-013.

This research article is available in Journal of Marine Science and Technology: https://jmstt.ntou.edu.tw/journal/ 


\title{
LAMINAR FILM CONDENSATION ON A HORIZONTAL PLATE IN A POROUS MEDIUM WITH SURFACE TENSION EFFECTS
}

\author{
Tong-Bou Chang
}

Key words: surface tension, laminar film condensation, porous medium.

\begin{abstract}
The problem of steady filmwise condensation on a finite-size horizontal plate embedded in a porous medium is studied for the case in which a cold plate faces upwards with a dry saturation vapor. Due to the effects of surface tension forces, there is a two-phase zone lying between the liquid film and the vapor zone. As in classical filmwise condensation problems, it is assumed that; (a) the inertia within the liquid film is negligible, and (b) the properties of porous medium, dry vapor, and condensate are constant. A dimensionless parameter $B o_{c}$ is introduced to characterize liquid flow due to surface tension forces. The mean Nusselt number $\overline{N u}$ can be enhanced by increasing the surface tension parameter $B o_{c}$. Without considering the surface tension effects, a closed form correlation for the Nusselt number is also obtained in the present study.
\end{abstract}

\section{INTRODUCTION}

Heat transfer in a porous medium with phase change has received increasing attention due to its wide range of heat transfer applications. For example, the problems of the horizontal two-phase flow in a porous medium involving phase change have important applications in heat pipe design, geothermal energy utilization and thermal enhancement of oil recovery. When there exists a two-phase flow in a porous medium, it is known that Darcy's law is applicable to both the liquid and the vapor phases.

The classical analysis of laminar film condensation on vertical or inclined surfaces was first performed by Nusselt [15]. In that analysis, the condensate film was assumed to be thin; convective and inertial effects were negligible. Within the condensate film, the temperature profile is linear. After the publication of Nusselt's work in 1916, many other researchers had extended the analyses by removing the overly restricted

Paper Submitted 04/26/05, Accepted 06/30/05. Author for Correspondence: Tong-Bou Chang. E-mail: tbchang@mail.stut.edu.tw.

*Assistant Professor, Department of Mechanical Engineering, Southern Taiwan University of Technology, 1, Nai-Tai Street, YungKang City, Tainan County, Taiwan, R.O.C. assumptions $[2,8,9,17,19]$, a review for these analyses can be found in Merte's work [12]. The earliest attempt to consider the condensation heat transfer rate on a horizontal surface was experimentally done by Popov [16] in 1951. Gerstmann and Griffith [5] then investigated the condensation on the underside of a horizontal plate both theoretically and experimentally. The case of the upper side condensation of a horizontal plate was first studied by Leppert and Nimmo [10] and Nimmo and Leppert [14]. However, they left one point in argument. The film condensation thickness at the plate edge is either assumed or specified by the particular boundary condition. Shigechi et al. [18] obtained the condensate thickness and heat transfer results on a horizontal plate by adjusting the inclined angle of the vapor-liquid interface at the plate edge. Yang and Chen [22] used the concept of minimum mechanical energy [1] to search the boundary condition at the horizontal plate edge.

The problem of a downward condensate flowing along a cool vertical or inclined surface in the presence of a porous medium has been extensively studied $[3,4$, 6]. Recently, Wang et al. [21] reveal that the condensation heat transfer coefficient can be enhanced by increasing values of the wave number and amplitude of the horizontal wavy surface. Most of the previous theoretical investigations do not consider the effect of surface tension forces. In many of these problems, the effective pore radii are small, thereby accentuating the significance of surface tension effects. Udell [20] investigated the heat and mass transfer characteristics of a sand-water-system by including surface tension forces. Majumdar and Tien [11] studied the effect of surface tension on film condensation on a vertical wall.

The problem of laminar film condensation on a horizontal plate in a porous medium is investigated in the present study with the consideration of surface tension effects. In order to achieve the goal of finding the effects of surface tension forces, the present study used the concepts of the thermodynamics of the twophase zone to analyze the liquid flow driven by the surface tension forces. Beside, the concept of minimum 
mechanical energy is applied to obtain the minimum film thickness at the plate edge. The dimensionless heat transfer coefficient can thus be properly solved for different values of Darcy number $D a$, Jakob number $J a$, effective Rayleigh number $R a$, effective Prandtl number $\operatorname{Pr}_{e}$ and the surface tension parameter $B o_{c}$.

\section{ANALYSIS}

Consider a finite-size horizontal flat plate with wall temperature $T_{w}$ embedded in a porous medium filled with a dry vapor. The vapor is pure and at a uniform temperature $T_{\text {sat }}$. If the wall temperature $T_{w}$ is lower than the saturation temperature $T_{s a t}$, and the liquid wets the plate surface ideally, a film of condensate will be formed on the plate surface. Adjacent to this film, there will be a region where the vapor and the liquid will co-exist to form a two-phase zone. Beyond this region a vapor zone will exist. A schematic diagram of the physical model and coordinate system is shown in Figure 1.

Under steady-state conditions, the liquid film boundary layer will be established with maximum depth existing at the center of plate and gradually decreasing to minimum depth at the plate edge. The analysis of film condensation about a horizontal flat plate in a porous medium with surface tension effects is developed under the following assumptions.

1. The flow is steady and laminar.

2. The inertia within the film is negligible (a creeping film flow is assumed).

3. The wall temperature and vapor temperature are uniform and are kept constant.

4. The kinetic energy of the film flow is negligible.

5. Darcy's law is applicable to the liquid film in the porous medium.

6 . The properties of porous medium, dry vapor, and condensate are kept constant.

Under the aforementioned assumptions, the governing equations in the liquid film with boundary layer simplifications are given as

Continuity equation:

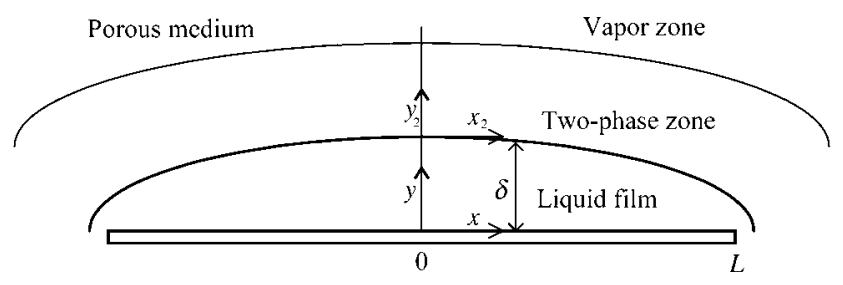

Fig. 1. Condensate film flow on a finite-size horizontal plate with porous medium.

$$
\frac{\partial u}{\partial x}+\frac{\partial v}{\partial y}=0
$$

The momentum equation along $x$-direction:

$$
0=-\frac{\partial P}{\partial x}-\frac{\mu_{e}}{K} u
$$

The momentum equation along $y$-direction:

$$
0=-\frac{\partial P}{\partial y}-\rho g
$$

Energy equation:

$$
u \frac{\partial T}{\partial x}+v \frac{\partial T}{\partial y}=\alpha_{e} \frac{\partial^{2} T}{\partial y^{2}}
$$

where $K$ is the intrinsic permeability of the porous medium, $u$ and $v$ are the Darcian velocity components in the $x$ - and $y$-directions, respectively, $P$ is the pressure of liquid, $\delta$ is the liquid film thickness, and $\mu_{e}$ and $\alpha_{e}$ are the effective dynamic viscosity and the effective thermal diffusivity of a porous medium saturated with liquid, respectively.

The static pressure $P$ can be obtained by integrating Eq. (3) with the use of boundary condition $P=P_{\text {sat }}$ at the interface of the two-phase zone with the liquid film $(y=\delta)$ gives,

$$
P=P_{\text {sat }}+\rho g(\delta-y)
$$

Substituting Eq. (5) into Eq. (2) yields

$$
u=-\frac{\rho g K}{\mu_{e}} \frac{d \delta}{d x}
$$

At the interface of the two-phase zone with the liquid film, the mass continuity equation is given as

$$
\left.\frac{\partial u_{2}}{\partial x}\right|_{y_{2}=0}+\left.\frac{\partial v_{2}}{\partial y_{2}}\right|_{y_{2}=0}=0
$$

where subscript 2 represent the properties in the twophase zone and $y_{2}=0$ corresponds to the interface of the two-phase zone with the liquid film.

At this interface, the no slip condition can be written as

$$
\left.u_{2}\right|_{y_{2}=0}=\left.u\right|_{y=\delta}
$$

or

$$
\begin{aligned}
& \left.\frac{\partial u_{2}}{\partial x}\right|_{y_{2}=0}=\left.\frac{\partial u}{\partial x}\right|_{y=\delta} \\
& =-\frac{\rho g K}{\mu_{e}} \frac{d^{2} \delta}{d x^{2}}
\end{aligned}
$$


Using the concepts of capillary pressure [20], the velocity in the two-phase zone can be written in the following form.

$$
\begin{aligned}
& u_{2}=\frac{K K_{r 1}}{\mu_{e}} \frac{\partial P_{c}}{\partial x} \\
& v_{2}=\frac{K K_{r 1}}{\mu_{e}} \frac{\partial P_{c}}{\partial y_{2}}
\end{aligned}
$$

where

$$
\begin{aligned}
& K_{r 1}=s^{3} \\
& P_{c}=\frac{\sigma}{\sqrt{K / \varepsilon}} f(s) \\
& f(s)=1.417(1-s)-2.120(1-s)^{2}+1.263(1-s)^{3}
\end{aligned}
$$

$s$ is the dimensionless saturation and $s=1$ at $y_{2}=0$

Substituting Eqs. (11a)-(11d) into Eq. (10), the capillary suction velocity of the two-phase zone $v_{2}$ can be expressed as

$$
v_{2}=\frac{K}{\mu_{e}} s^{3}\left(\frac{\sigma}{\sqrt{K / \varepsilon}} f^{\prime} \frac{\partial s}{\partial y_{2}}\right)
$$

Substituting Eqs. (8b) and (12) into Eq. (7), Eq. (7) is transformed into the following form.

$$
\begin{aligned}
& -\frac{\rho g K}{\mu_{e}} \frac{d^{2} \delta}{d x^{2}}+\frac{K}{\mu_{e}} \frac{\sigma}{\sqrt{K / \varepsilon}}\left[s^{3} f^{\prime \prime}\left(\frac{\partial s}{\partial y_{2}}\right)^{2}\right. \\
& \left.+3 s^{2} f^{\prime}\left(\frac{\partial s}{\partial y_{2}}\right)^{2}+f^{\prime} s^{3} \frac{\partial^{2} s}{\partial y_{2}^{2}}\right]\left.\right|_{y_{2}=0}=0
\end{aligned}
$$

According to the results Majumdar and Tein [11], the saturation profiles near the interface of the twophase are almost linear. It can be expressed as at

$$
\frac{\partial^{2} s}{\partial y_{2}^{2}}=0 \text { at } y_{2}=0
$$

Eq. (13) can thus be written as

$$
\left.\frac{\partial s}{\partial y_{2}}\right|_{y_{2}=0}=-\sqrt{\frac{-1}{\left.\left(s^{3} f^{\prime \prime}+3 s^{2} f^{\prime}\right)\right|_{y_{2}=0} \frac{\sqrt{K / \varepsilon}}{\sigma} \times \rho g \frac{d^{2} \delta}{d x^{2}}}}
$$

Substituting Eq. (15) into Eq. (12), the velocity of the liquid which leaves the liquid film into the twophase zone via the surface tension forces can thus be solved as follows

$$
\left.v_{2}\right|_{y_{2}=0}=\left.\frac{K}{\mu_{e}}\left(\left(\frac{s^{3} f^{\prime}}{s^{3} f^{\prime \prime}+3 s^{2} f^{\prime}}\right) \sqrt{\frac{-\rho g \sigma}{\sqrt{K / \varepsilon}} \times \frac{d^{2} \delta}{d x^{2}}}\right)\right|_{y_{2}=0}
$$

Using Nusselt's classical analysis method, the overall energy balance in the liquid film can be written as

$$
\begin{aligned}
& \frac{d}{d x}\left\{\int_{0}^{\delta} \rho u\left(h_{f g}+C p\left(T_{s}-T\right)\right) d y\right\} d x \\
& +\left.\rho h_{f g} v_{2}\right|_{y_{2}=0} d x=\frac{k_{e} \Delta T}{\delta} d x
\end{aligned}
$$

where $k_{e}$ is the effective thermal conductivity of a porous medium saturated with liquid.

The right hand side of Eq. (17) represents the energy transfer from liquid film to solid plate, the first term of the left hand side as the net energy flux across the liquid film (from $x$ to $x+d x$ ), and the second term of the left hand side as the net energy transferred into the two-phase zone. Substituting Eqs. (6) and (16) into Eq. (17), yields

$$
\begin{aligned}
& \delta \frac{d}{d x}\left(\delta \frac{d \delta}{d x}\right)-\delta\left(\frac{h_{f g}}{h_{f g}+\frac{1}{2} C p \Delta T}\right)\left(\left(\frac{s^{3} f^{\prime}}{s^{3} f^{\prime \prime}+3 s^{2} f^{\prime}}\right)\right. \\
& \left.\sqrt{\frac{-\sigma}{\rho g \sqrt{K / \varepsilon}} \times \frac{d^{2} \delta}{d x^{2}}}\right)\left.\right|_{y_{2}=0} \\
& =\frac{-k_{e} \Delta T \mu_{e}}{\rho^{2} g K\left(h_{f g}+\frac{1}{2} C p \Delta T\right)}
\end{aligned}
$$

Eq. (18) can be simplified by defining the following dimensionless parameters

$$
\begin{aligned}
& J a=\frac{C p \Delta T}{h_{f g}+\frac{1}{2} C p \Delta T} \\
& R a=\frac{\rho^{2} g \operatorname{Pr}_{e} L^{3}}{\mu_{e}^{2}}
\end{aligned}
$$




$$
\begin{aligned}
& \operatorname{Pr}_{e}=\frac{\mu_{e} C p}{k_{e}} \\
& D a=\frac{K}{L^{2}} \\
& B o_{c}=\frac{\sigma \sqrt{\varepsilon}}{\rho g K}=\frac{1}{B o}
\end{aligned}
$$

The $B o_{c}$ number is a ratio of surface tension force and gravity force. If the $B o_{c}$ number becomes larger (a smaller Bond number), then the surface tension forces are dominated and hence the contribution of the twophase zone to the total liquid flow. Eq. (18) can be rewritten as:

$$
\begin{aligned}
& \delta \frac{d}{d x}\left(\delta \frac{d \delta}{d x}\right)-\left.\delta\left(1-\frac{1}{2} J a\right)\left(\frac{s^{3} f^{\prime}}{s^{3} f^{\prime}+3 s^{2} f^{\prime}}\right)\right|_{y_{2}=0} \\
& \times \sqrt{\left(-B o_{c} \sqrt{K} \frac{d^{2} \delta}{d x^{2}}\right)}=\frac{-J a}{R a_{e}} \frac{L}{D a}
\end{aligned}
$$

with the following boundary conditions:

$$
\begin{aligned}
& \frac{d \delta}{d x}=0 \text { at } x=0 \\
& \delta=\delta_{\min } \text { at } x=L
\end{aligned}
$$

With these boundary conditions, we still cannot solve Eq. (20), since $\delta_{\min }$ is unknown.

Obviously, the film thickness at the plate edge cannot be zero, in fact, it should be established by the application of a minimum mechanical energy principle $[1,22]$. The principle states that a fluid flowing across a hydrostatic pressure gradient and off the plate will adjust itself so that the rate of mechanical energy within the fluid will be minimal with respect to the boundary layer at the plate edge. The minimum (critical) thickness can thus be calculated by setting the derivative of mechanical energy with respect to $\delta$ equal to zero for the steady flow rate;

$$
\left.\left[\frac{\partial}{\partial \delta} \int_{0}^{\delta}\left(\frac{u^{2}}{2}+g y+\frac{P}{\rho}\right) \rho u d y\right]\right|_{\dot{m}_{c}}=0
$$

where is the critical value of mass flow out of the plate edge.

Substituting Eqs. (5) and (6) into Eq. (23) yields the new boundary condition

$$
\left.\frac{d \delta}{d x}\right|_{L}=-\left(\frac{\mu_{e}^{2}}{\rho^{2} g K^{2}} \delta_{\min }\right)^{1 / 2}
$$

By introducing the normalized variables of $x^{*}=x / \sqrt{K}, \delta^{*}=\delta / \sqrt{K}$, for Eq. (20), which can be written as

$$
\begin{aligned}
& \delta^{*} \frac{d}{d x}\left(\delta^{*} \frac{d \delta^{*}}{d x^{*}}\right)-\left.\left(1-\frac{1}{2} J a\right)\left(\frac{s^{3} f^{\prime}}{s^{3} f^{\prime \prime}+3 s^{2} f^{\prime}}\right)\right|_{y_{2}=0} \\
& \times \delta^{*} \sqrt{\left(-B o_{c} \frac{d^{2} \delta^{*}}{d x^{* 2}}\right)} \\
& =\frac{-J a}{R a D a^{3 / 2}}
\end{aligned}
$$

Eq. (21) becomes at

$$
\frac{d \delta^{*}}{d x^{*}}=0 \text { at } x^{*}=0
$$

and Eq. (24) becomes

$$
\left.\frac{d \delta^{*}}{d x^{*}}\right|_{x_{L}^{*}}=-\left(\frac{\operatorname{Pr}_{e}}{R a D a^{3 / 2}} \delta_{\min }^{*}\right)^{-1 / 2}
$$

From Eqs. (25) and (11c) and the corresponding boundary conditions Eqs. (26a) and (26b), we can obtain the dimensionless film thickness $\delta^{*}$ in terms of $J a$, $R a, \mathrm{Pr}_{e}, B o_{c}$, and $\mathrm{Da}$.

Eq. (25) is solved numerically by using RungeKutta shooting method to obtain the dimensionless liquid film thickness. Since the slope of the dimensionless liquid film thickness is not flat at the plate edge, the progressively finer grid size toward the plate edge is used in the calculations.

The mean Nusselt number is also calculated as

$$
\overline{N u}=\frac{\bar{h} L}{K_{e}}
$$

where

$$
\bar{h}=\frac{1}{L} \int_{0}^{L} h(x) d x=\frac{1}{L} \int_{0}^{L} h \frac{k_{e}}{\delta} d x=\frac{1}{L} \int_{0}^{x_{L}^{*}} \frac{k_{e}}{\delta^{*}} d x^{*}
$$

\section{RESULTS AND DISCUSSION}

At first, the effects of surface tension on the film condensation in a porous medium are neglected. In the other words, the surface tension parameter $B o_{c}$ is set to zero. Then Eq. (25) becomes

$$
\delta^{*} \frac{d}{d x^{*}}\left(\delta^{*} \frac{d \delta^{*}}{d x^{*}}\right)=\frac{-J a}{R a_{e} D a^{3 / 2}}
$$


The above equation and its corresponding boundary conditions can be solved by the same numerical procedures mentioned in the previous section, and the resulting $\delta^{*}$ value along $x^{*}$-direction can be obtained.

Figure 2 shows that the calculated Nusselt number decreases when $\mathrm{Ja} / \mathrm{Ra}$ increases. In fact, the relationship is almost linear and can be expressed as

$$
\overline{N u} /\left(D a^{0.32} R a^{0.32} \mathrm{Ja}^{-0.35}\right)=1.231-0.02(\mathrm{Ja} / \mathrm{Pr})
$$

for $D a<10^{-2}$ and $J a / \operatorname{Pr}<0.1$.

The maximum difference between the numerical results and the results of Eq. (30) is less than $2 \%$. Beside, the constraint of $D a=K / L^{2}<10^{-2}$ in the above equation is usually satisfied for common porous materials, because the permeability $K$ of many practically porous materials is at the order of (see Table 1.1 in reference [13] or Table 2.3 in reference [7]). For water vapor condensing on a smooth plate, the value of is much less than 0.1 and is about 0.0025 at one atmosphere. The resulting Nusselt number, Eq. (30), then becomes

$$
\overline{N u}=1.23\left(\mathrm{Da}^{0.32} \mathrm{Ra}^{0.32} \mathrm{Ja}^{-0.35}\right)
$$

Wang et al. [21] used a new transformation method to investigate the film condensation on a horizontal wavy plate in a porous medium but without the consideration of surface tension. Table 1 shows the comparison of Wang's results of wave number $n=0$ (con-

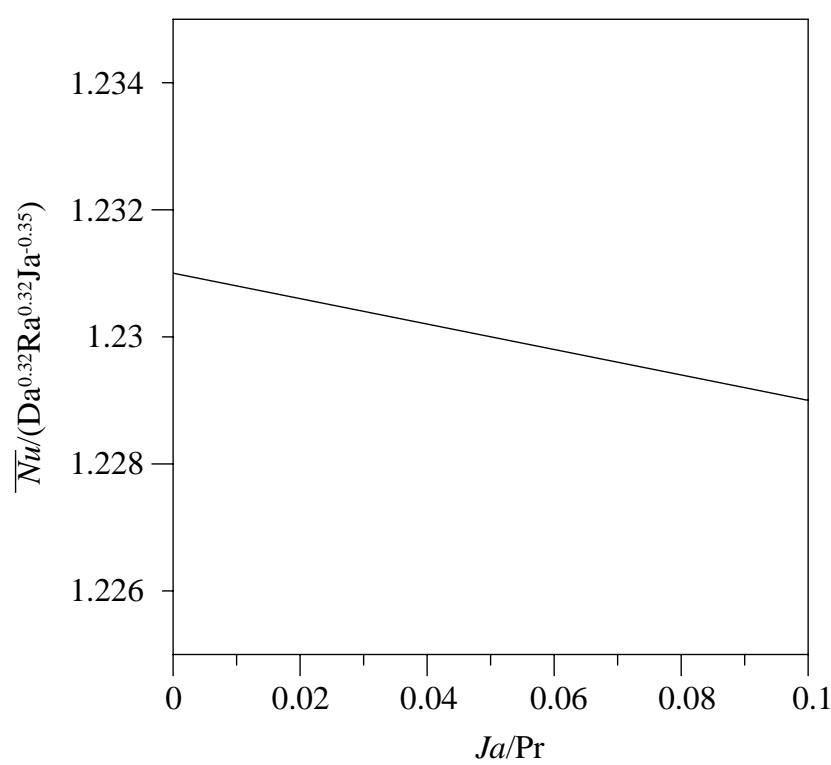

Fig. 2. Dimensionless heat transfer coefficient without surface tension effects. densation on a flat plat) and the present study. It can be estimated that the maximum difference is lower than $1.6 \%$ between the solutions by Wang et al. [21] and those by the present study.

Figure 3 shows that the Nusselt number increases upon increasing $B o_{c}$. A special dashed line is added in this figure to distinguish whether the effects of surface tension can be ignored or not. At the left side of the dashed line, the effects of surface tension are small and can be neglected. This is due to the small value of which means that the effect of surface tension is small, so that the $\mathrm{Bo}_{c}$ will be close to the resulting of Eq. (31). But at the right side of the dashed line, it is seen that the values of increase with $B o_{c}$ at different values of $\operatorname{Pr}, D a$, $J a$, and $R a$. The physical reason underlying this is that higher values of $B o_{c}$ imply stronger surface tension forces, which result more liquid condensate into the two-phase zone. This creates a thinner liquid film and a sharper temperature gradient in the liquid film, which result in a higher heat transfer rate.

Figures 4(a) and 4(b) show the profile distribu-

Table 1. Comparison of the solutions by Wang et al. [21] and those by the present study

\begin{tabular}{ccc}
\hline Parameters & $\overline{N u}$ (Wang et al.) & $\overline{N u}$ (Present study) \\
\hline$J a=0.01, R a=10^{5}$, & & \\
$D a=0.01, \operatorname{Pr}=7$. & 55.7 & 56.2 \\
\hline$J a=0.1, R a=10^{5}$, & & \\
$D a=0.01, \operatorname{Pr}=7$. & 25.5 & 25.1 \\
\hline
\end{tabular}

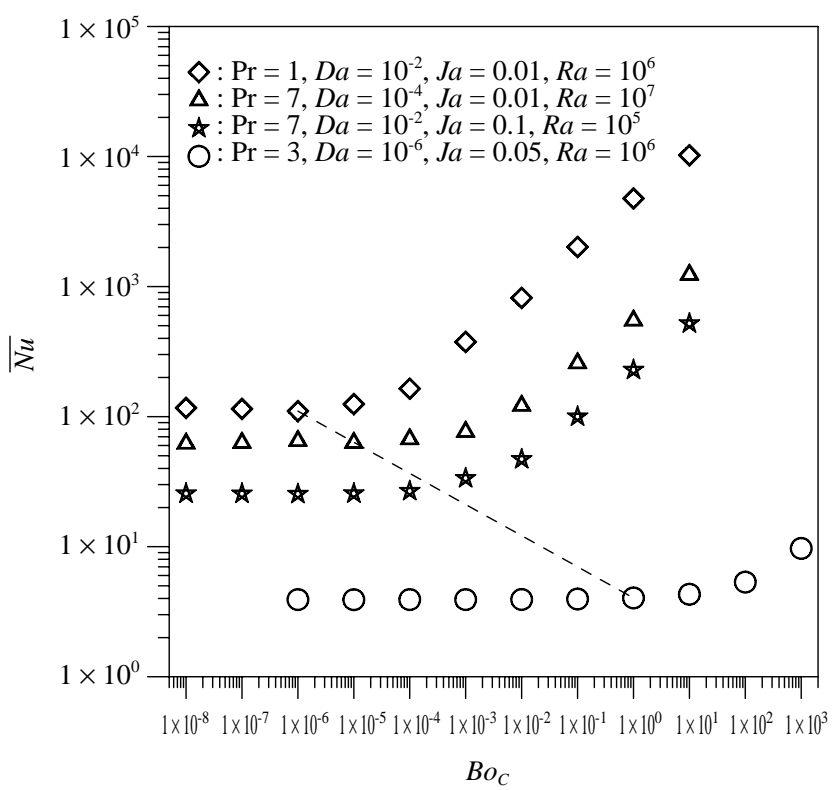

Fig. 3. Dimensionless heat transfer coefficient with surface tension effects. 
tions of the dimensionless liquid film thickness ratio $\delta /$ $\delta_{0}$ for $\operatorname{Pr}=0.7, D a=10^{-4}, J a=0.01, R a=10^{7}$ and $\operatorname{Pr}=$ 7, $D a=10^{-2}, J a=0.1, R a=10^{5}$, respectively. From these two figures we can see that an increase in $B o_{c}$ decreases the dimensionless liquid film thickness ratio $\delta / \delta_{0}$. Besides, the difference of $\delta / \delta_{0}$ along the plate is the largest at the plate edge $\left(x * / x^{*}{ }_{L}=1\right)$ between any two different values of $B o_{c}$. That is because the liquid

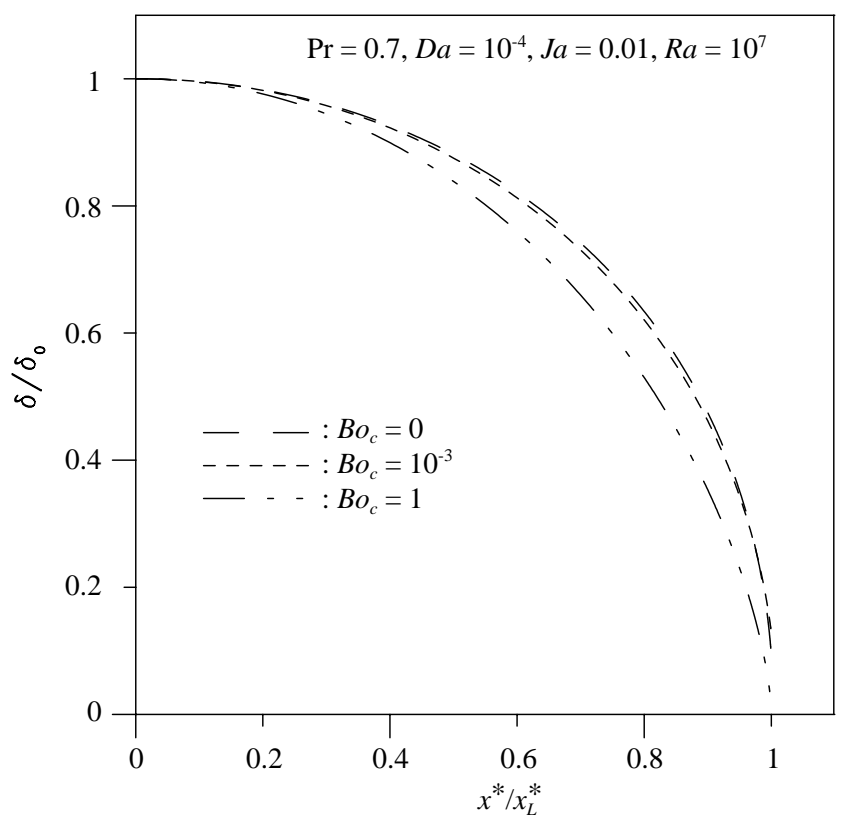

(a)

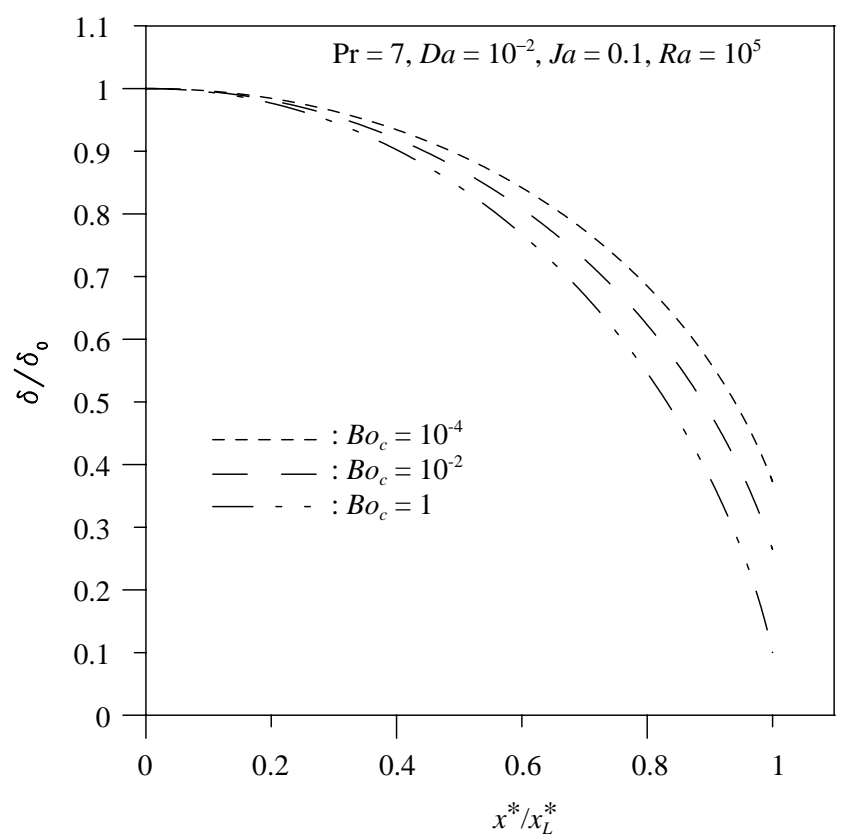

(b)

Fig. 4. Dimensionless liquid film thickness ratio for (a) $\operatorname{Pr}=0.7, D a=$ $10^{-4}, J a=0.01, R a=10^{7}$; (b) $\operatorname{Pr}=7, D a=10^{-2}, J a=0.1, \operatorname{Ra}=10^{5}$.

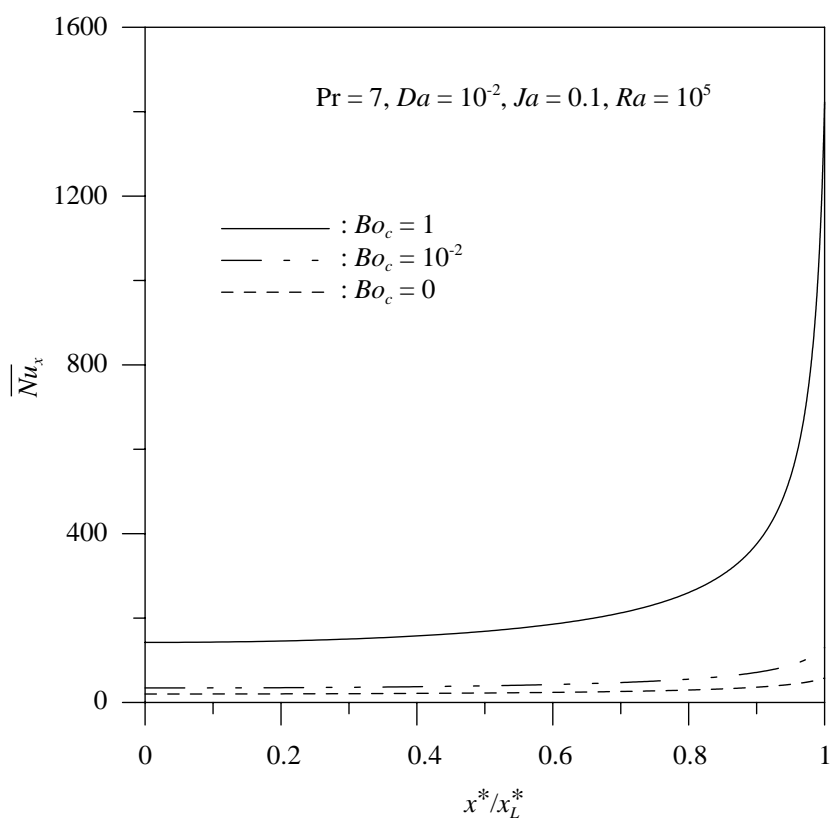

Fig. 5. The local Nusselt number distribution $N u_{x}$ with variation of $B o_{c}$.

film thickness is decreased from the plate center to the plate edge. For the purpose of sucking the liquid condensate from the liquid film into the two-phase zone, the effects of surface tension will become relatively larger for a thinner liquid film thickness.

The influence of $B o_{c}$ on the local Nusselt number $N u_{x}$ for $\operatorname{Pr}=7, D a=10^{-2}, J a=0.1, R a=10^{5}$ is displayed in Figure 5. From this figure, it can be seen that the value of increases from the plate center to the plate edge. That is because the liquid film thickness is decreased from the plate center to the plate edge, so that the local thermal resistance decreases as the liquid film becomes thinner. In addition, from this figure we also can see that the value of $N u_{x}$ increases with the increase of $B o_{c}$. On the other hand, the effect of $B o_{c}$ on the $N u_{x}$ is maximum at the plate edge. The reason is the same as mentioned before since the liquid film thickness is minimum at the plate edge, the effects of surface tension will become larger for a thinner liquid film thickness.

\section{CONCLUSION}

The problem of laminar film condensation on a horizontal plate in a porous medium with surface tension effects is performed in the present study. With the presence of surface tension forces, there will be a twophase zone just above the liquid film. A dimensionless parameter $B o_{c}$ is introduced to characterize liquid flow driven by the surface tension forces. The heat transfer performance can thus be enhanced by the capillary 
suction of the two-phase zone, and it is observed that a higher value of $B o_{c}$ will induce a higher heat transfer performance and a lower liquid film thickness. A closed form correlation for the Nusselt number of the case without the consideration of surface tension forces is also be obtained in the present study.

\section{ACKNOWLEDGMENTS}

This work was supported by the National Science Council of ROC: NSC93-2212-E-218-013.

\section{NOMENCLATURE}

$B o_{c}$ ratio of surface tension and gravity forces defined in Eq. (19e)

Cp Specific heat at constant pressure

Da Darcy number defined in Eq. (19d)

$g \quad$ acceleration of gravity

$h \quad$ heat transfer coefficient

$h_{f g} \quad$ heat of vaporization

$\mathrm{Ja}$ Jakob number defined in Eq. (19a)

$k \quad$ thermal conductivity

$K \quad$ permeability of the porous medium

$L \quad$ half of plate width condensate mass flux

$\mathrm{Nu} \quad$ Nusselt number defined in Eq. (27)

$P \quad$ pressure

$\operatorname{Pr}_{e} \quad$ effective Prandtl number

$R a$ effective Rayleigh number

$s \quad$ dimensionless saturation

$T$ temperature

$\Delta T \quad$ saturation temperature minus wall temperature

$u, v$ horizontal and vertical velocity component

\section{Geek symbols}

$\delta \quad$ condensate film thickness

$\delta_{0} \quad$ condensate film thickness at plate center

$\mu \quad$ liquid viscosity

$\rho \quad$ liquid density

$\alpha \quad$ thermal diffusivity

$\sigma \quad$ surface tension

$\varepsilon \quad$ porosity

\section{Superscripts}

indicates average quantity

indicates dimensionless variable

\section{Subscripts}

2 properties in the two-phase zone

$o \quad$ quantity at plate center

c capillary

min minimum quantity or quantity at plate edge

sat quantity at wall effective property

\section{REFERENCES}

1. Bakhmeteff, B.K., Hydraulics of Open Channel, McGraw-Hill, New York, pp. 39-41 (1966).

2. Chen, M.M., "An Analytical Study of Laminar Film Condensation: Part 1-Flat Plates," J. Heat Tran., Vol. 83, pp. 48-54 (1961).

3. Cheng, P., "Film Condensation Along an Inclined Surface in a Porous Medium," Int. J. Heat Mass Tran., Vol. 24, No. 6, pp. 983-990 (1981).

4. Cheng, P. and Chui, D.K., "Transient Film Condensation on a Vertical Surface in a Porous Medium," Int. J. Heat Mass Tran., Vol. 27, pp. 795-798 (1984).

5. Gerstmann, J. and Griffith, P., "Laminar Film Condensation on the Underside of Horizontal and Inclined Surfaces," Int. J. Heat Mass Tran., Vol. 10, No. 2, pp. 567-580 (1976).

6. Kaviany, M., "Boundary Layer Treatment of Film Condensation in the Presence of a Solid Matrix," Int. J. Heat Mass Tran., Vol. 29, pp. 951-954 (1986).

7. Kaviany, M, Principles of Heat Transfer in Porous Media, Springer-Verlag, New York, pp. 26 (1991).

8. Koh, J.C.Y., "On Integral of Treatment of Two Phase Boundary Layer in Flim Condensation," J. Heat Transf., Vol. 83, pp. 359-362 (1961).

9. Koh, J.C.Y., Sparrow, E.M., and Hartnett, J.P., "The Two Phase Boundary Layer in Laminar Film Condensation," Int. J. Heat Mass. Tran., Vol. 2, pp. 69-82 (1961).

10. Leppert, G. and Nimmo, B., "Laminar Film Condensation on Surface Normal to Body or Inertial Forces," $J$. Heat Tran., Vol. 80, No. 1, pp. 178-179 (1968).

11. Majumdar, A. and Tein, C.L., "Effects of Surface Tension on Film Condensation in a Porous Medium," J. Heat Trans -T. ASME, Vol. 112, pp. 751-757 (1990).

12. Merte Jr., H., "Condensation Heat Transfer," Adv. Heat Tran., Vol. 9, pp. 181-272 (1973).

13. Nield, D.A. and Bejan, A., Convection in Porous Media, Springer-Verlag, New York, p. 4 (1992).

14. Nimmo, B. and Leppert, G., "Laminar Film Condensation on a Finite Horizontal Surface," Proceedings of $4^{\text {th }}$ International Heat Transfer Conference, pp. 402-403 (1970).

15. Nusselt, W., "Die Oberflachen Kondensation des Wasserdampes," Zeitsehrift des Vereines Deutscher Ingenieure, Vol. 60, No. 2, pp. 541-546 (1916).

16. Popov, V.D., "Heat Transfer During Vapor Condensation on a Horizontal Surfaces," Trudy Kiev. Teknol. Inst. Pishch, Prom. Vol. 11, No. 1, pp. 87-97 (1951).

17. Rohsenow, W.M., "Heat Transfer and Temperature Distribution in Laminar Film Condensation," J. Heat Trans 
-T. ASME, Vol. 78, pp. 1645-1648 (1956).

18. Shigechi, T., Kawae, N., Tokita, Y., and Yamada, T., "Film Condensation Heat Transfer on a Finite-Size Horizontal Plate Facing Upward," JSME Series B, Vol. 56, No. 1, pp. 205-210 (1990).

19. Sparrow, E.M. and Gregg, J.L., "Laminar Condensation Heat Transfer on a Horizontal Cylinder," J. Heat Trans -T. ASME, Vol. 81, pp. 291-296 (1959).

20. Udell, K.S., "Heat Transfer in Porous Media Heated From Above With Evaporation, Condensation, and Cap- illary Effects," J. Heat Trans -T. ASME, Vol. 105, pp. 485-492 (1983).

21. Wang, S.C., Chen, C.K., and Yang, Y.T., "Film Condensation on a Finite-Size Horizontal Plate Bounded by a Homogenous Porous Layer," Appl. Therm. Eng., Vol. 25, pp. 577-590 (2005).

22. Yang, S.A. and Chen, C.K., "Laminar Film Condensation on A Finite-Size Horizontal Plate with Suction at Wall," Appl. Math. Model., Vol. 16, No. 1, pp. 325-329 (1992). 